\title{
On strongly normal tesselations
}

\author{
Peter Brass* \\ Institut für Informatik, FU Berlin \\ Takustrasse 9, D-14195 Berlin, Germany
}

\begin{abstract}
A tesselation $\mathcal{C}$ is called strongly normal, if it is normal (topological discs with intersections that are either empty or connected) and for any subset of cells $C_{1}, \ldots, C_{k}, C^{*}$ of the tesselation holds: if the intersection $\bigcap_{i=1}^{k} C_{i}$ of all $C_{i}$ is nonempty and each $C_{i}$ has nonempty intersection with $C^{*}$, then the intersection $C^{*} \cap \bigcap_{i=1}^{k} C_{i}$ of all $C_{i}$ with $C^{*}$ is nonempty. This concept was introduced for polygonal or polyhedral cells in a recent paper by Saha and Rosenfeld, where they proved that it is equivalent to the topological property that any cell together with any set of neighboring cells forms a simply connected set. Answering a question from their paper, it is shown here that at least in the plane the cells need not be convex polygons, but can be arbitrary topological discs. Also the property is already implied if all collections of three cells have this property, giving a simpler characterization and a connection to Helly-type theorems.
\end{abstract}

\section{Introduction}

One possible interpretation of digital geometry is that it models some region of image space by a finite set of cells $\mathcal{C}$ (corresponding to the possible image points), and studies properties of subsets of $\mathcal{C}$ (the image) like convexity, connectedness etc. Normally the underlying tesselation is the regular tesselation by squares, but there has also been some work on regular hexagonal or triangular tesselations, as well as on arbitrary tesselations. It is reasonable to restrict our study to normal tesselations (e.g. Grünbaum and Shephard, 1987), i.e. collections of cells such that each cell is a topological disk, two cells have no interior points in common, the intersection of two cells, if nonempty, is connected, and the cells are uniformly bounded in size (this last condition is necessary in classical tiling theory, but trivially satisfied in our situation, where the number of cells is finite. We do not require the union of the cells to cover the plane.) Tesselations by convex polygons (polyhedra) are always normal in this classic (tiling-theory) sense; Saha and Rosenfeld (1998) required a slightly stronger property: that the polyhedral tiling should be faceto-face, but this is not necessary.

In a recent paper, Saha and Rosenfeld (1998) introduced the concept of strong normality, a neighbourhood intersection condition which they showed to be equivalent to a local topological well-behavedness condition. A set $\mathcal{C}$ of cells of a tesselation is strongly normal, if it is normal and for each $C^{*}, C_{1}, \ldots, C_{k} \in \mathcal{C}$ if $\bigcap_{i=1}^{k} C_{i} \neq \emptyset$ and $C^{*} \cap C_{i} \neq \emptyset$ $(i=1, \ldots, k)$, then $C^{*} \cap \bigcap_{i=1}^{k} C_{i} \neq \emptyset$. Saha and Rosenfeld showed that for normal tesselations by polygons or polyhedra this is equivalent to the property that for each $C^{*}, C_{1}, \ldots, C_{k} \in \mathcal{C}$ if $C^{*} \cap C_{i} \neq \emptyset(i=1, \ldots, k)$, then $C^{*} \cup \bigcup_{i=1}^{k} C_{i}$ is simply connected. Thus if any intersection of neighbours of $C^{*}$, if notempty, meets $C^{*}$, then any union of $C^{*}$ with some neighbours is simply connected. Some tesselations have this property, e.g. the regular square and hexagonal tesselation are strongly normal; others, like the regular

* e-mail brass@inf.fu-berlin.de 
triangular tesselation, are not strongly normal: for if $T_{1}, T_{2}, T_{3}$ are edge-to-edge neighbours of a central triangle $T$, then $T_{2}, T_{3}$ are neighbours of $T_{1}$, but the union $T_{1} \cup T_{2} \cup T_{3}$ is not simply connected.

At the end of their paper, Saha and Rosenfeld asked whether their result on tesselations by polygons and polyhedra could be generalized to arbitrary (normal) tesselations. It is the aim of this paper to provide that generalization.

\section{The twodimensional case}

The main result of this paper is

Theorem: Let $\mathcal{C}$ be a normal tesselation. The following properties of $\mathcal{C}$ are equivalent:

(1) If $C^{*}, C_{1}, \ldots, C_{k} \in \mathcal{C}$ with $\bigcap_{i=1}^{k} C_{i} \neq \emptyset$ and $C^{*} \cap C_{i} \neq \emptyset$ for each $i$, then $C^{*} \cap \bigcap_{i=1}^{k} C_{i} \neq \emptyset$.

(2) If $A, B, C \in \mathcal{C}$ with $A \cap B \neq \emptyset, A \cap C \neq \emptyset, B \cap C \neq \emptyset$ then $A \cap B \cap C \neq \emptyset$.

(3) If $A, B_{1}, B_{2} \in \mathcal{C}$ with $A \cap B_{1} \neq \emptyset, A \cap B_{2} \neq \emptyset$ then $A \cup B_{1} \cup B_{2}$ is simply connected.

(4) If $C^{*}, C_{1}, \ldots, C_{k} \in \mathcal{C}$ with $C^{*} \cap C_{i} \neq \emptyset$ for each $i$, then $C^{*} \cup \bigcup_{i=1}^{k} C_{i}$ is simply connected.

Proof: $(1) \Rightarrow(2)$ is trivial, since (2) is just the special case $k=2$ of (1).

$(2) \Rightarrow(1)$ is a consequence of the topological Helly theorem (Helly 1930; Molnár, 1957; Eckhoff, 1993):

Theorem: (topological Helly theorem) Let $\mathcal{D}$ be a finite family of connected, simply connected sets in the plane, such that any two of them have connected intersection, and any three have nonempty intersection, then all of them have nonempty intersection.

To deduce now (1) from (2), we take the set $\left\{C^{*}, C_{1}, \ldots, C_{k}\right\}$ as $\mathcal{D}$ and note that the pairwise intersections are nonempty by the assumption of (1), and connected, since $\mathcal{C}$ is a normal tesselation. The intersection of any three of them is nonempty by the assumption of (1), if $C^{*}$ is not among the three sets, and by an application of (2) to the assumption of (1), if $C^{*}$ is among three sets. Thus we can apply the above theorem and obtain that the intersection of all sets in $\left\{C^{*}, C_{1}, \ldots, C_{k}\right\}$ is nonempty, which is the conclusion of (1). $(2) \Rightarrow(3)$ Let $A, B_{1}, B_{2} \in \mathcal{C}$ be three cells with $A \cap B_{1} \neq \emptyset, A \cap B_{2} \neq \emptyset$, and let $\gamma$ be a closed curve in $A \cup B_{1} \cup B_{2}$ which we wish to contract to a point. Assume first that $A \cap B_{1} \cap B_{2}$ is not empty, and $w$ a point from this intersection. The curve $\gamma$ consists of intervals in which it is in a single set $\left(A\right.$ or $B_{1}$ or $\left.B_{2}\right)$, and from each such interval we introduce an arc from the curve through the set to $w$ and back to the curve (growing these loops is a homotopy). Then we have a new curve which consists of many loops, starting at $w$, going through one of our sets, reaching $\gamma$ and following it into another set, then returning to $w$. Each loop is therefore a closed curve that goes only through the union of two of our sets, two topological discs with connected intersection, so each loop can be independently contracted to $w$.

It remains the case that $A \cap B_{1} \cap B_{2}$ is empty; then by property (2) the intersection $B_{1} \cap B_{2}$ is empty. Thus any closed curve $\gamma$ that goes through all three sets cannot go from $B_{1}$ 
directly to $B_{2}$, but has to go through $A$. Thus $\gamma$ can be decomposed into arcs starting in $A$, going through $B_{i}$ and returning to $A$, and each of these arcs can independently contracted until he is in $A$, then the whole curve in $A$ can be contacted to a point. So $A \cup B_{1} \cup B_{2}$ is simply connected.

$(3) \Rightarrow(2)$ Let $A, B, C \in \mathcal{C}$ be three cells with pairwise nonempty intersection, and suppose that $A \cap B \cap C$ is empty. We will show that then $A \cup B \cup C$ is not simply connected, contradicting (3). For this we select a closed curve $\gamma$ which starts in some point $a \in A$, goes to some point $b \in B$, then to $c \in C$, and returns to $a$. We can select $\gamma$ in such a way that it is the boundary of a topological disc $X$. We now use the following lemma (Lyusternik, 1963), which follows from Sperner's lemma and is known as an important step in the proof of Brouwer's fixed-point theorem:

Lemma: Let $X$ be a topological disk, $p_{1}, p_{2}, p_{3}$ points on the boundary of $X$, and $Y_{1}, Y_{2}, Y_{3}$ three closed sets such that $X \subseteq Y_{1} \cup Y_{2} \cup Y_{3}, p_{i} \in Y_{i}$, and the boundary of $X$ between $p_{i}$ and $p_{i+1}$ is covered by $Y_{i} \cup Y_{i+1}$ for $i=1,2,3$. Then there is a point $q \in X$ such that $q \in Y_{1} \cap Y_{2} \cap Y_{3}$.

Using $a, b, c$ as $p_{1}, p_{2}, p_{3}$ and $A, B, C$ as $Y_{1}, Y_{2}, Y_{3}$ we find that if $X \subseteq A \cup B \cup C$, then $A \cap B \cap C$ is nonempty, contradicting our assumption. Thus there is a point $r \in X \backslash$ $(A \cup B \cup C$ ), and since $A, B, C$ are closed sets, there is a whole disk around $r$ that does not belong to $A \cup B \cup C$. Now the winding number of $\gamma$ around $r$ is one, and this is the winding number of any homotopic image of $\gamma$, as long as the path of the homotopy is in $A \cup B \cup C$ (does move across $r$ ). So $\gamma$ cannot be contracted to a point (with winding number zero), contradicting our assumption (3).

$(3) \Rightarrow(4)$ Let $C^{*}, C_{1}, \ldots, C_{k} \in \mathcal{C}$ such that $C^{*} \cap C_{i} \neq \emptyset$, and let $\gamma$ be a closed curve in $C^{*} \cup C_{1} \cup \cdots \cup C_{k}$. To contract $\gamma$ to a point, we select a point $w$ in $C^{*}$, partition $\gamma$ in intervals such that each interval is in a single set from $\left\{C^{*}, C_{1}, \ldots, C_{k}\right\}$, and introduce in each interval a loop from $\gamma$ through the set and $C_{r}$ to $w$, and back to $\gamma$. Then we can repartition the curve into intervals starting and ending at $w$. Each of these $w$-loops is a closed curve going through $C^{*}$ and at most two of the $C_{i}$. Thus by (3) each loop can be contracted independently to the point $w$. This proves (4).

$(4) \Rightarrow(3)$ finally is again trivial, (3) being the special case $k=2$ of (4).

\section{Further Remarks}

The threedimensional problem is probably more difficult, since the only known higherdimensional variants of the topological Helly theorem require either algebraic topology (Helly (1930): if $\mathcal{F}$ is a family of homology cells in $\mathbb{R}^{d}$ such that the intersection of any $k \leq d$ of them is again a homology cell, and the intersection of any $d+1$ is nonempty, then the intersection of all of them is nonempty), or stronger intersection conditions (Matoušek 1995).

For convex cells we have the classical Helly theorem (a finite collection of $d$-dimensional convex sets has nonempty intersection iff each subcollection of $d+1$ sets has), so we can state that it is sufficient to check the intersection condition only for $k \leq d$ neighbouring sets $C_{1}, \ldots, C_{k}$ instead of for arbitrary numbers $k$.

Saha e.a. (1997) studied strongly normal tetrahedralizations of spatial domains. This is probably less useful, since any strongly normal subdivision of some spatial domain $X$ 
into polyhedral cells must be such that for each interior cell $C$ and each face $F$ of $C$ there is another (opposite) face $F^{\prime}$ of $C$ disjoint to $F$ (cubical cells being the smallest such example). For if $C$ is an interior cell with face $F$, and each of the other $C$-faces has a common point with $F$, then one can select three other faces $F_{1}, F_{2}, F_{3}$ of $C$ such that each of these faces has a nonempty intersection with $F$, and $F_{1} \cap F_{2} \cap F_{3} \neq \emptyset$, but $F \cap F_{1} \cap F_{2} \cap F_{3}=\emptyset$. So the cells belonging to these faces on the other side of $C$ do not satisfy the intersection condition of strong normality.

Thus it is simple to construct strongly normal tetrahedralizations of any convex or starlike domain: just select one point in the interior, triangulate the boundary, and join each boundary triangle to the interior point to obtain a tetrahedron. But there are no useful strongly normal tetrahedralizations, since we cannot make the tetrahedra small. So cubical space-divisions are the simplest type of 'arbitrarily fine' strongly normal cell divisions.

\section{References}

Eckhoff, J, 1993. Helly, Radon and Carathéodory Type Theorems, 389-445 in Handbook of Convex Geometry Vol A, P.M. Gruber and J.M. Wills, Eds., Elsevier/North Holland Grünbaum, B., Shephard, G.C., 1987. Tilings and Patterns, W.H. Freeman, New York

Helly, E., 1930. Über Systeme abgeschlossener Mengen mit gemeinschaftlichen Punkten, Monatsh. Math. 37 281-302

Lyusternik, L.A., 1963. Convex Figures and Polyhedra, (translated from the Russian by T. Jefferson Smith) Dover Publications, New York

Matoušek, J., 1995. A Helly-type theorem for unions of convex sets, Proc. Eleventh Annual Symposium on Computational Geometry 1995, 138-146, ACM Press

Molnár, J., 1957. Über den zweidimensionalen topologischen Satz von Helly (in Hungarian, with Russian and German summary) Mat. Lapok 8 108-114

Saha, P.K., Rosenfeld, A., 1998. Strongly normal sets of polygons and polyhedra, Pattern recognition letters 19 1119-1124

Saha, P.K., Dutta Majumder, D., Rosenfeld, A., 1998. Local topological parameters in a tetrahedral representation, Graphical Models and Image Processing Vol. 60 423-436 http://www . cfar . umd.edu/ftp/TRs/CVL-Reports-1997/TR3826-saha.ps.gz 